\title{
Two Patients with Dry Eye Disease Followed Up Using an Expression Assay of Ocular Surface Mucin
}

\author{
Yumiko Machida Jun Shoji Natsuko Harada Noriko Inada \\ Division of Ophthalmology, Department of Visual Sciences, Nihon University School of \\ Medicine, Tokyo, Japan
}

\section{Key Words}

Dry eye $\cdot$ Rebamipide $\cdot$ Fluorometholone $\cdot$ Mucin $\cdot$ MUC5AC $\cdot$ MUC16

\begin{abstract}
Purpose: We report 2 patients with dry eye disease followed up using the expression levels of ocular surface mucin. Case Reports: Patient 1: a 57-year-old woman with Sjögren's syndrome-associated dry eyes experienced severe dryness and foreign body sensation in both her eyes, and instilled sodium hyaluronate ophthalmic solution $0.3 \%$ about $10-15$ times daily. We measured the expression levels of MUC5AC mRNA (MUC5AC) and MUC16 mRNA (MUC16) by using real-time reversed transcription polymerase chain reaction for the specimens of modified impression cytology. Expression levels of MUC5AC and MUC16 on her ocular surface were very low. Subjective symptoms and expression levels of ocular surface mucin improved after combined treatment of rebamipide (4 times daily) and fluorometholone (once daily) ophthalmic suspension. Patient 2: a 62-year-old man with chronic graftversus-host disease-associated dry eye experienced severe foreign body sensation and developed superficial punctate keratopathy with mucous thread and filamentary keratitis. Expression level of MUC5AC was very high at baseline. Subjective symptoms and expression levels of ocular surface mucin improved by combined treatment of rebamipide (4 times daily) and fluorometholone (once daily) ophthalmic suspension. Conclusion: Clinical test for MUC gene expression on the ocular surface was found to be useful in the follow-up of dry eye treatment.


Machida et al.: Two Patients with Dry Eye Disease Followed Up Using an Expression Assay of Ocular Surface Mucin

\section{Introduction}

Sjögren's syndrome is a chronic and systemic autoimmune disease characterized by lymphocytic infiltration of the exocrine glands [1]. The clinical characteristics of Sjögren's syndrome are dry eye and dry mouth. The ocular complications of graft-versus-host disease (GVHD) caused by rejection after bone marrow transplantation are characterized by pseudomembranous conjunctivitis in the acute phase and dry eye in the chronic phase [2]. These dry eyes belong to the type aqueous-deficient dry eyes and present with similar clinical observations such as objective findings, including ocular dryness, discomfort, and ocular pain, and subjective symptoms, including papillary hyperplasia with velvety appearance and superficial punctate keratitis (SPK) with mucous discharge.

Recently, a novel medical treatment using mucin-related and mucosal protective ophthalmic suspension, rebamipide (Mucosta ${ }^{\circledR}$ ophthalmic suspension UD 2\%, Ostuka Pharmaceutical, Co., Ltd.), has become available for the treatment of the dry eye diseases in practice. A multicenter, open-label, 52 -week study of $2 \%$ rebamipide ophthalmic suspension in patients with dry eye showed its effectiveness in improving both the objective signs and subjective symptoms of dry eye patients [3]. Alternatively, anti-inflammatory ophthalmic solutions including cyclosporine and steroid are reportedly effective for the treatment of dry eye disease $[4,5]$. However, the alteration of ocular surface mucin during the treatment of dry eye disease it is still not well understood.

To assess the effectiveness of combined treatment with rebamipide and steroid ophthalmic suspension for dry eye patients, we treated two patients, one with Sjögren's syndrome-associated dry eye (SS-DE) and another with chronic GVHD-associated dry eye (cGVHD-DE), with rebamipide and steroid ophthalmic suspensions and monitored their gene expression levels of ocular surface mucin.

\section{Case Report}

Methods of Gene Expression Assay for Ocular Surface Mucin

Clinical tests for gene expression of the ocular surface mucin were approved by the institutional review board of Nihon University Itabashi Hospital. These clinical tests aimed to investigate mucin core protein gene (MUC) expression in the human ocular surface of healthy individuals and patients with dry eye in vivo by using Shirmer's test paper.

Tips of the Shirmer's test papers were applied to the upper tarsal conjunctiva and then removed. The Shirmer's test paper was then preserved in RNAlater RNA Stabilization Reagent (Qiagen, Hilden, Germany) until analysis. For detection of $M U C$ expression by real-time reversed transcription polymerase chain reaction (real-time RT-PCR), total RNA from each specimen was harvested using an RNeasy ${ }^{\circledR}$ Mini Kit (Qiagen) per the manufacturer's manual. cDNA was then synthesized using a High-Capacity cDNA Reverse Transcription Kit (Life Technologies Japan, Tokyo, Japan) according to the manufacturer's instructions. Real-time RT-PCR was performed using TaqMan gene expression assay (Life Technologies Japan) and predesigned primers (Life Technologies Japan): MUC5AC (Hs00873651_m1), and MUC16 (Hs01065189_m1). Samples were analyzed using a real-time PCR system (Step One Plus ${ }^{\mathrm{TM}}$; Life Technologies Japan), and comparative threshold $(\mathrm{Ct})$ values were obtained. Data were analyzed by the $\triangle \triangle C T$ method. The normal levels of the MUC5AC and MUC16 considered the results of the eight healthy volunteers. 
Machida et al.: Two Patients with Dry Eye Disease Followed Up Using an Expression Assay of Ocular Surface Mucin

The expression levels [median (range)] of MUC5AC and MUC16 on the ocular surface of eight healthy normal volunteers $(2$ men, 6 women; mean age \pm standard deviation: $46.0 \pm$ 19.8 years) were $1.02(0.10-4.45)$ and $0.15(0.05-0.58)$, respectively.

\section{Clinical Course of the Patients}

Patient 1

A 57-year-old woman was diagnosed with SS-DE and pemphigus vulgaris at another hospital and had been experiencing severe subjective symptoms such as dryness, ocular pain, and foreign body sensation in both eyes. Although she was treated with sodium hyaluronate (SH) ophthalmic solution $0.3 \%$ (Hyalein ${ }^{\circledR}$ mini, Santen Pharmaceutical Co., Ltd., Osa$\mathrm{ka}$, Japan) and artificial tears, her symptoms did not improve at all. She performed frequent instillation of the SH ophthalmic solution 10-15 times daily because of severe symptoms.

In April 2014, she visited the Department of Ophthalmology, Nihon University Itabashi Hospital. The Dry Eye-related Quality of life Score (DEQS) was used to assess the subjective score of the patient [6]. The DEQS at the baseline observational point and 5 weeks after initial treatment was 93.3 and 88.3, respectively. Slit-lamp examination of objective findings of ocular surface showed conjunctival swelling with follicular formation in the upper palpebral conjunctiva (fig. 1a) and SPK with fluorescein staining in the lower mid-peripheral cornea (fig. 1b). Blepharitis with skin erosion caused by pemphigus vulgaris was also observed (fig. 1c), and blinking imperfection might thereby occur. Abnormalities of tear dynamics were shown by Schirmer's test values of $3 \mathrm{~mm}$ in right eye and $5 \mathrm{~mm}$ in left eye, and tear brake-up time (TBUT) of $7 \mathrm{~s}$ in right eye and $8 \mathrm{~s}$ in left eye. The fluorescein staining was scored as follows. The ocular surface was divided into three areas (nasal conjunctiva, cornea, and temporal conjunctiva) that were assessed for fluorescein staining. Each area was judged a staining score from 0 (no damage) to 3 (severe damage) points, and the total score of fluorescein staining was calculated ranging from 0 to 9 points. Fluorescein scores of the right and left eyes were 1 and 1 point, respectively.

The patient was treated with a combination of rebamipide ophthalmic suspension four times daily and fluorometholone ophthalmic suspension (Flumetholon ${ }^{\circledR}$ ophthalmic suspension $0.1 \%$, Santen Pharmaceutical Co., Ltd.) once daily for dry eye. As for the SH instillation, frequent instillation was changed to twice-daily instillation after the initial presentation. Blepharitis was treated with dexamethasone ophthalmic ointment (Santezon ${ }^{\circledR}$ ophthalmic ointment $0.05 \%$, Santen Pharmaceutical Co., Ltd.). After having been treated with dexamethasone ophthalmic ointment for 1 week, the blepharitis treatment was continued using white petroleum gel (PROPETO ${ }^{\circledR}$; Maruishi Pharmaceutical; Tokyo, Japan).

The Schirmer's test value, TBUT, and fluorescein score did not improve after 3 months with combined treatment for dry eye (fig. 1d). However, her subjective symptoms including ocular pain improved dramatically; DEQS score (93.3 to 88.3) and expression levels of ocular surface mucin also improved (shown in fig. 1e, f). MUC5AC at baseline point showed an extremely low level and was restored by the combined treatment to the levels comparable to those in healthy subjects. MUC16 also gradually increased by these treatments.

Patient 2

A 62-year-old man with a clinical diagnosis of cGVHD-DE had undergone related-donor bone marrow transplantation for acute myelogenous leukemia in 2011 and experienced irritation and foreign body sensation in both eyes. He was treated with ophthalmic administration including rebamipide ophthalmic suspension four times daily, methylprednisolone containing fradiomycin sulfate (Neo-Medrol ${ }^{\circledR}$ EE Ointment, Pfizer Co., Ltd., Tokyo, Japan) 
Machida et al.: Two Patients with Dry Eye Disease Followed Up Using an Expression Assay of Ocular Surface Mucin

once daily, and betamethasone sodium phosphate containing fradiomycin sulfate (Rinderon $^{\circledR}$-A, Shionogi Pharmaceutical Co., Ltd., Osaka, Japan) 3 times daily. However, his symptoms gradually worsened despite treatment, and he was referred to us. Slit-lamp examination showed SPK with mucous thread and filamentary keratitis (fig. 2). Abnormalities of tear dynamics were shown by Schirmer's test values of $4 \mathrm{~mm}$ in the right eye and $10 \mathrm{~mm}$ in the left eye, and TBUT of $1 \mathrm{~s}$ in the right eye and $1 \mathrm{~s}$ in the left eye. The patient's treatment was changed to combined treatment of rebamipide ophthalmic suspension four times daily and fluorometholone ophthalmic suspension once daily. The SH ophthalmic solution was used at any time, if necessary. Subjective symptoms related with cGVHD-DE and corneal epithelial damage improved after change of prescription. DEQS score was restored to 26.7 from 51.2. SPK with mucous thread and filamentary keratitis of his left eye disappeared at 1 month after the combined treatment. SPK with mucous thread and filamentary keratitis of his right eye also disappeared at 3 months after the combined treatment. The condition of both eyes had subsided after 5 months.

MUC5AC and MUC16 at baseline point showed high-level MUC5AC and low-level MUC16, and they were restored by combined treatment to levels comparable to those in healthy subjects. Considering the relationship between $M U C$ expression on ocular surface including MUC5AC and MUC16 and ocular findings with fluorescein staining, MUC expression showed high levels when SPK with mucous thread and filamentary keratitis persisted, whereas MUC expression levels were restored to the same levels as those in healthy subjects when their clinical findings disappeared.

\section{Discussion}

Herein, we report the cases of two patients with dry eye disease, in whom we were able to confirm the efficacy of the combined treatment with rebamipide and steroid ophthalmic suspension by monitoring the expression levels of the ocular surface mucin. The clinical characteristics before the combined treatment in these patients are as follows: patient 1 with SS-DE had severe subjective symptoms due to frequent instillation of SH ophthalmic solution, and expression of MUC5AC on her ocular surface decreased dramatically; patient 2 with cGVHD-DE had keratoconjunctival epithelial damage with mucous thread and filamentary keratitis, and expression of $M U C 5 A C$ on his ocular surface increased significantly.

The characteristics of patient 1 showed that SS-DE was caused by MUC5AC decrease; in addition to the pathologic condition of SS-DE, frequent instillation was believed to be responsible for reducing MUC5AC expression on the ocular surface. Combined treatment with rebamipide and fluorometholone ophthalmic suspension improved the subjective symptoms of the patients and increased expression levels of MUC5AC and MUC16 to the levels same as those in healthy subjects. The MUC gene expression assay on the ocular surface is a novel clinical test, which may help understand the severity of subjective symptoms in patients with dry eyes.

The rebamipide ophthalmic suspension reportedly has pharmacological effects such as induction of mucin production and the anti-inflammatory effect [7]. On the other hand, antiinflammatory ophthalmic solution, such as cyclosporine A was reportedly effective for treating inflammation of the ocular surface and keratoconjunctival epithelial damages in SS-DE [4]. Furthermore, the steroid ophthalmic solutions are reportedly effective as therapeutic drugs for moderate to severe dry eye patients [5]. The representative problems of steroid ophthalmic solution in dry eye treatment were the exacerbation of the epithelial damage in ocular surface and the steroid-induced glaucoma owing to long-term use. Moreover, steroid 
Machida et al.: Two Patients with Dry Eye Disease Followed Up Using an Expression Assay of Ocular Surface Mucin

instillation treatment was successful for the treatment of dry eye because we selected lowdose steroid that did not increase intraocular pressure and excluded dry eye patients with glaucoma or contraindication to steroids [8]. Because the use of cyclosporine ophthalmic solution was not approved for treatment of dry eyes in Japan, we used steroid ophthalmic solution for this combination therapy. In our two patients, fluorometholone ophthalmic suspension instillation once daily may have a great effect without adverse effect for the longterm treatment of dry eye disease. The MUC16 expression on ocular surface continued to increase gradually during the long-term treatment. The membranous mucin such as MUC16 is thought to become the index of the epithelial damage of the ocular surface. Therefore, the epithelial damage of the ocular surface was thought to improve gradually by this combined treatment. At short-term follow-up of this combined treatment, MUC5AC indicated a low response or overreaction according to the severity of inflammation and epithelial damage in the ocular surface. However, when the degree of the inflammation and the epithelial damage in the ocular surface were relieved by long-term combined treatment, the increase of the MUC16 expression became slight, and the MUC5AC expression was thought to be maintained at the same level as that in healthy subjects. Improvement of the mucin expression on the ocular surface was thought to be one of the reasons why subjective symptoms became milder. However, whether there is a difference between monotherapy and combined treatment needs to be investigated in a larger sample group.

Alternatively, the characteristics of patient 2 showed that cGVHD-DE was a MUC5ACincreased type dry eye, and the SPK with the mucous thread was shown as an objective finding of ocular surface inflammation. In patients with cGVHD-DE, the expression levels of $M U C 5 A C$ were controversial. In our case, expression of MUC5AC on ocular surface was high before the combined treatment. In severe cGVHD-DE, MUC5AC expression on the ocular surface decreased, and this alteration was restored by cyclosporine treatment [8]. However, the pathophysiology of severe inflammation and mild epithelial damage in the ocular surface of the patient with cGVHD-DE was thought to increase the production of MUC5AC from the goblet cells in the conjunctiva. Furthermore, short-term combined treatment of rebamipide and steroid ophthalmic suspension may increase expression of MUC5AC, and regression of ocular surface inflammation with the long-term combined treatment might maintain the MUC5AC expression level similar to that in a healthy subject.

In our case study, patients with dry eye disease were thought to have a couple of clinical stages of low-level expression or high-level expression of MUC5AC on the ocular surface. Further, a normal range of $M U C 5 A C$ expression levels is observed in the healthy subjects, and the combined treatment for dry eye may converge to the normal expression levels of $M U C 5 A C$ in patients with dry eye disease. However, it is difficult to define the normal range definitely at present. In the future, therapeutic drugs for treating dry eye may be chosen depending on the expression levels of ocular surface mucin.

In conclusion, examination of ocular surface mucin was found to be useful in the followup of dry eye treatment. Combined treatment of rebamipide and steroid ophthalmic suspension was effective for improving subjective symptoms and stabilizing the expression of ocular surface mucin.

\section{Statement of Ethics}

The clinical tests for gene expression of the ocular surface mucin were approved by the institutional review board of Nihon University Itabashi Hospital. Informed consent was obtained from the patients. 
Machida et al.: Two Patients with Dry Eye Disease Followed Up Using an Expression Assay of Ocular Surface Mucin

\section{Disclosure Statement}

None of the authors have any conflict of interest with the submission of this case report, and no financial support was received for this paper.

\section{References}

-1 Foulks GN, Forstot SL, Donshik PC, Forstot JZ, Goldstein MH, Lemp MA, Nelson JD, Nichols KK, Pflugfelder SC, Tanzer JM, Asbell P, Hammitt K, Jacobs DS: Clinical guidelines for management of dry eye associated with Sjögren disease. Ocul Surf 2015;13:118-132.

-2 Espana EM, Shah S, Santhiago MR, Singh AD: Graft versus host disease: clinical evaluation, diagnosis and management. Graefes Arch Clin Exp Ophthalmol 2013;251:1257-1266.

-3 Kinoshita S, Awamura S, Nakamichi N, Suzuki H, Oshiden K, Yokoi N; Rebamipide Ophthalmic Suspension Long-term Study Group: A multicenter, open-label, 52-week study of 2\% rebamipide (OPC-12759) ophthalmic suspension in patients with dry eye. Am J Ophthalmol 2014;157:576-583.

4 Zhou XQ, Wei RL: Topical cyclosporine A in the treatment of dry eye: a systematic review and meta-analysis Cornea 2014;33:760-767.

5 Avunduk AM, Avunduk MC, Varnell ED, Kaufman HE: The comparison of efficacies of topical corticosteroids and nonsteroidal anti-inflammatory drops on dry eye patients: a clinical and immunocytochemical study. Am J Ophthalmol 2003;136:593-602.

-6 Sakane Y, Yamaguchi M, Yokoi N, Uchino M, Dogru M, Oishi T, Ohashi Y, Ohashi Y: Development and validation of the Dry Eye-Related Quality-of-Life Score questionnaire. JAMA Ophthalmol 2013;131:13311338.

7 Kimura K, Morita Y, Orita T, Haruta J, Takeji Y, Sonoda KH: Protection of human corneal epithelial cells from TNF- $\alpha$-induced disruption of barrier function by rebamipide. Invest Opthalmol Vis Sci 2013;54:2572-2760.

-8 Jung HH, Ji YS, Sung MS, Kim KK, Yoon KC: Long-term outcome of treatment with topical corticosteroids for severe dry eye associated with Sjögren's syndrome. Chonnam Med J 2015;51:26-32. 
Case Reports in

Ophthalmology

Machida et al.: Two Patients with Dry Eye Disease Followed Up Using an Expression Assay of Ocular Surface Mucin

a

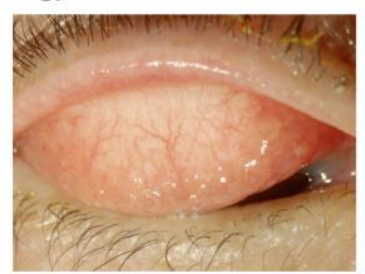

b

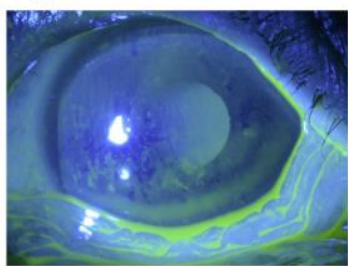

C

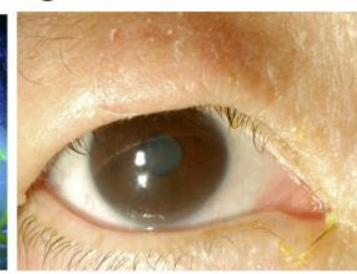

d

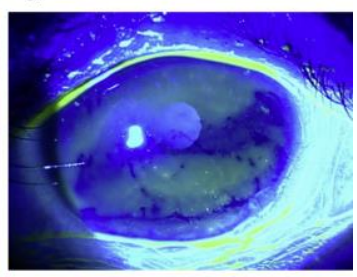

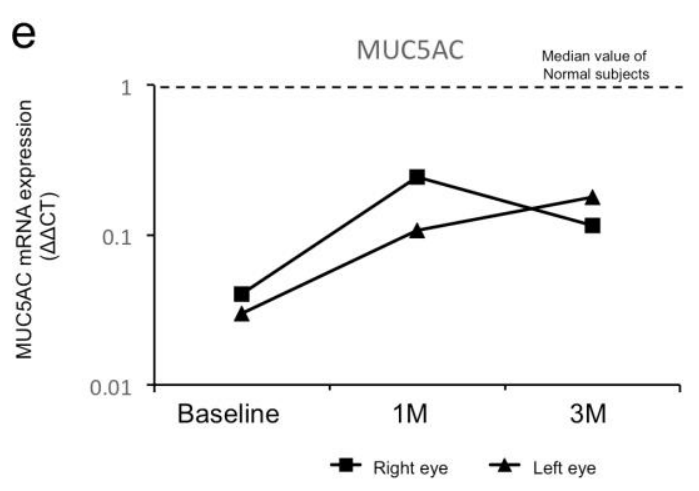

f

MUC16

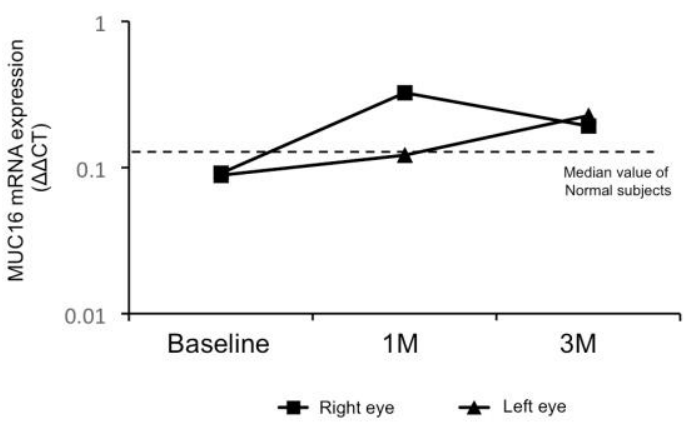

Fig. 1. Slit-lamp photograph and mucin mRNA expression on the ocular surface of patient 1. a Slit-lamp photograph of the upper tarsal conjunctiva the right eye showing follicular formation at initial treatment (baseline). b Slit-lamp photograph of the cornea of the right eye showing mild fluorescein staining at initial treatment (baseline). c Photograph of the right eye lid showing swelling and skin erosion at initial treatment (baseline). $\mathbf{d}$ Slit-lamp photograph of the cornea of the right eye showing mild fluorescein staining at 3 months after treatment. e Course of MUC5AC mRNA expression on the ocular surface. $f$ Course of MUC16 mRNA expression on the ocular surface. $M=$ Month(s). 
Machida et al.: Two Patients with Dry Eye Disease Followed Up Using an Expression Assay of Ocular Surface Mucin

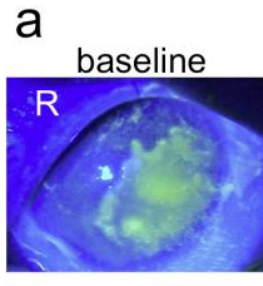

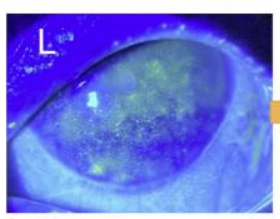

b

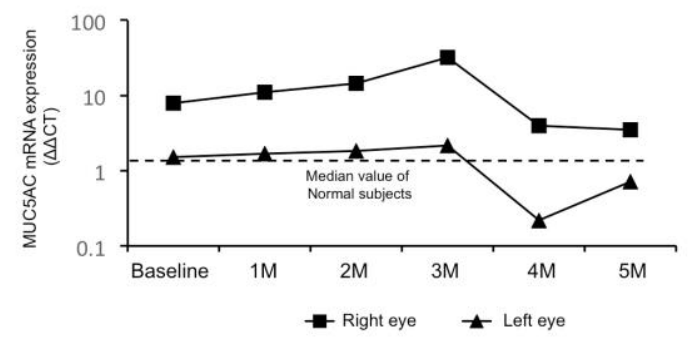

1 month
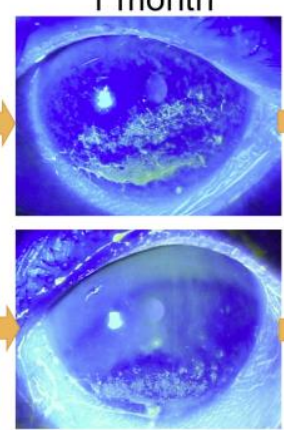

MUC5AC
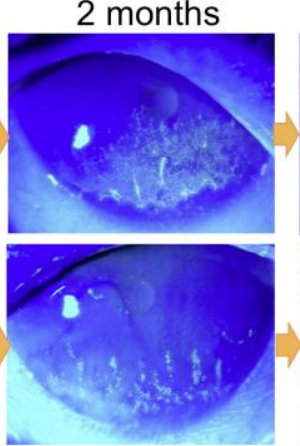

C
3 months
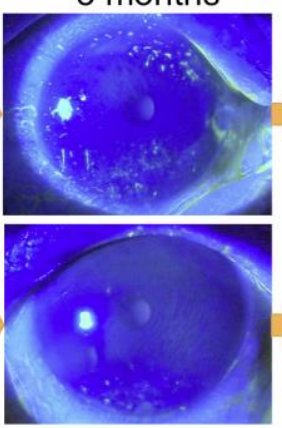

5 months
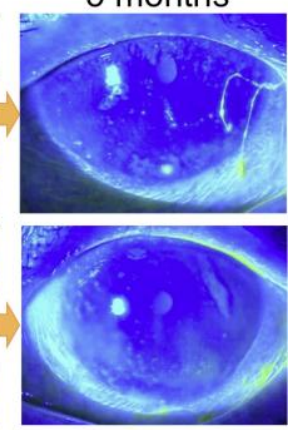

MUC16

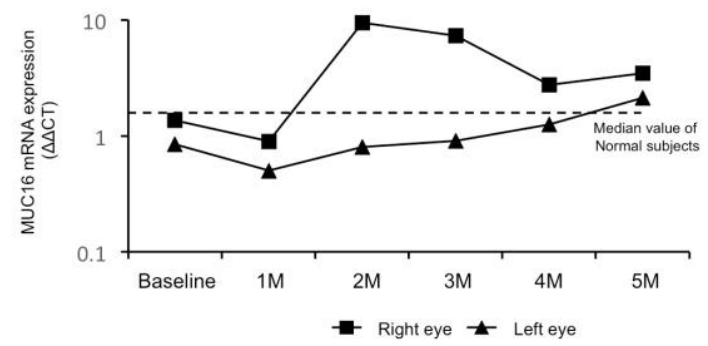

Fig. 2. Slit-lamp photographs and mucin mRNA expression on the ocular surface of patient 2. a Slit-lamp photographs of the right eye showing the course of corneal fluorescein staining. $\mathbf{b}$ Course of MUC5AC mRNA expression on the ocular surface. $c$ Course of MUC16 mRNA expression on the ocular surface. $\mathrm{M}=$ Month(s). 